\title{
Non-Hermitian Dirac Hamiltonian in Three-Dimensional Gravity and Pseudosupersymmetry
}

\author{
Özlem Yeşiltaş \\ Department of Physics, Faculty of Science, Gazi University, 06500 Ankara, Turkey \\ Correspondence should be addressed to Özlem Yeşiltaş; yesiltas@gazi.edu.tr
}

Received 13 August 2015; Accepted 4 November 2015

Academic Editor: Frank Filthaut

Copyright (C) 2015 Özlem Yeşiltaş. This is an open access article distributed under the Creative Commons Attribution License, which permits unrestricted use, distribution, and reproduction in any medium, provided the original work is properly cited. The publication of this article was funded by SCOAP $^{3}$.

\begin{abstract}
The Dirac Hamiltonian in the $(2+1)$-dimensional curved space-time has been studied with a metric for an expanding de Sitter space-time which is two spheres. The spectrum and the exact solutions of the time dependent non-Hermitian and angle dependent Hamiltonians are obtained in terms of the Jacobi and Romanovski polynomials. Hermitian equivalent of the Hamiltonian obtained from the Dirac equation is discussed in the frame of pseudo-Hermiticity. Furthermore, pseudosupersymmetric quantum mechanical techniques are expanded to a curved Dirac Hamiltonian and a partner curved Dirac Hamiltonian is generated. Using $\eta$-pseudo-Hermiticity, the intertwining operator connecting the non-Hermitian Hamiltonians to the Hermitian counterparts is found. We have obtained a new metric tensor related to the new Hamiltonian.
\end{abstract}

\section{Introduction}

Two great achievements of the twentieth century, quantum mechanics and general relativity, are very successful in their own boundaries to describe the nature; however, they are incompatible; for instance, they break down at extremely tiny distance which is Planck scale. In modern physics, the unified theory of gravitation and quantum mechanics which plays a fundamental role with exactly solvable gravitational field equations has always attracted considerable interest. On the other hand, experimental studies have been performed for the gravitational effects in quantum theory: earth's rotational effect on the phase of the neutron wave function [1], experimental nanodiamond interferometry [2], and quantum light in coupled interferometers [3]. In theoretical physics, interesting work including mathematical aspects of the Dirac Hamiltonians with their spectrum has been investigated such as shifted energy levels of the hydrogen atom in a region of curved space-time [4], modified supersymmetric harmonic oscillator on a two-dimensional gravitational field [5], exact solutions in a $(2+1)$-dimensional contracting and expanding curved space-time [6], singularities in $(2+1)$-dimensional space-time [7], and Hawking radiation of particles from a black hole [8]. Curved Dirac systems have also dynamical symmetries [9], considering that the $(2+1)$ dimensions $S L(2, c)$ symmetries of the Dirac Hamiltonian have been studied [5]. In nonrelativistic domain, $\mathscr{P} \mathscr{T}$ symmetric theories which are examining the non-Hermitian Hamiltonians with complex potentials and real eigenvalues have attracted much interest [10]. Here, $\mathscr{P}$ and $\mathscr{T}$ are the parity and time-reversal operators whose action on position wave functions $\psi(x)$ can be shown by $\mathscr{P} \psi(x)=\psi(-x)$ and $\mathscr{T} \psi(x)=\psi^{*}(x)$. More general concept of the Hermiticity is found as a generalization of the $\mathscr{P} \mathscr{T}$ symmetry which is called pseudo-Hermiticity [1113]. The real eigenvalues and corresponding eigenstates of a non-Hermitian Hamiltonian are associated with a symmetry such as the $\eta$-pseudo-Hermitian Hamiltonian. It is shown that any inner product may be defined in terms of a metric operator $\eta$. Moreover, hydrogen atom freely falling in a curved space-time with the curvature effects on the spectrum is investigated [4] and a general scalar product called the Parker product is defined for the Dirac equation in a curved background. It is also shown that if the time dependence of the metric is not omitted, there occurs a violation of 
Hermiticity in the Dirac Hamiltonian [4]. Then, in the light of a weight operator in Parker scalar product, a non-Hermitian Hamiltonian and its Hermiticity without omitting the time dependency of the metric are discussed in [14]. The problems of nonuniqueness and Hermiticity of Hamiltonians using the frame of pseudo-Hermitian Hamiltonian through the stationary gravitational fields and self-conjugacy of Dirac Hamiltonians with some examples are examined in $[15,16]$.

The low-dimensional systems such as $(2+1)$ dimensions may appear in the dynamics of charge carriers in graphene and some carbon nanostructures [17]. Our fundamental motivation for the Dirac equation in $(2+1)$-dimensional gravity may be a toy model for such systems. On the other hand, quantum mechanical effects of the gravitational field can be detected using the Dirac equation and its predictions in a curved space-time. These effects had been presaged by the nonrelativistic Schrodinger equation in the $\sim 1 / r$ potential but some corrections and the precision can be performed by the Dirac system in curved space-time. For instance, the energy level of one electron atom is shifted when it is put into a territory of curved space-time [4]. From this point of view, we use a different space-time metric in this study in order to see those effects. On the further side of these discussions, generalization of the supersymmetric quantum mechanics, that is, pseudosupersymmetric quantum mechanics and its effects in curved spacetime, has not been involved in the literature to our knowledge. Intertwining operators linking non-Hermitian Dirac Hamiltonians and Hermitian counterparts may give rise to more general Dirac Hamiltonian chains. Accordingly we have examined the Dirac equation in $(2+1)$-dimensional universe with an induced metric in this paper. We have given the real spectrum of the nonHermitian Dirac Hamiltonian and corresponding solutions of time dependent and angular parts. Moreover, we have shown that a new non-Hermitian Dirac Hamiltonian in curved space-time can be generated using the aspects of pseudosupersymmetric quantum mechanics and this may lead to another metric tensor which may be related to the generated Hamiltonian. This paper is organized as follows: Section 2 is devoted to the Dirac equation in $(2+1)$ curved space-time and separation of variables, Section 3 involves the exact solutions, and pseudosupersymmetric quantum mechanical applications are discussed in Section 4. Finally, we conclude the paper in Section 5.

\section{Dirac Equation}

The generally covariant form of the Dirac equation is

$$
i \gamma^{\mu}(x)\left(\partial_{\mu}+i e A_{\mu}-\Gamma_{\mu}(x)\right) \Psi(x)=M \Psi(x)
$$

where $M$ is the mass and $e$ is the charge of the particle, $A_{\mu}$ is the electromagnetic vector potential, $\Gamma_{\mu}(x)$ is the spin connection, and $\gamma^{\mu}(x)$ are the space-time dependent matrices. The spin connection relation is defined by

$$
\Gamma_{\mu}(x)=\frac{1}{4} g_{\lambda \rho}\left(e_{\nu, \mu}^{a} e_{a}^{\rho}-\Gamma_{\nu \mu}^{\rho}\right) S^{\lambda \nu}
$$

where $\Gamma_{\nu \mu}^{\rho}$ is the Christoffel symbol. In [18], the induced metric which is the static form of the Euclidean de Sitter space-time is given by

$$
d s^{2}=\ell^{2} d \tau^{2}-\ell^{2} \sinh ^{2} \tau d \theta^{2}-\ell^{2} \sinh ^{2} \tau \sin ^{2} \theta d \phi^{2}
$$

where $\tau \in[0, \infty), \phi \in[0,2 \pi)$, and $\theta \in[0, \pi)$ and $\ell$ is the radius of the universe. The vielbein matrix has become

$$
e_{a}^{\mu}(x)=\left(\begin{array}{ccc}
\frac{1}{\ell} & 0 & 0 \\
0 & \frac{1}{\ell \sinh \tau} & 0 \\
0 & 0 & \frac{1}{\ell \sinh \tau \sin \theta}
\end{array}\right)
$$

here $\mu$ labels the general space-time coordinate and $a$ labels the local Lorentz space-time. The vielbein field as the square root of the metric tensor is written as

$$
\begin{gathered}
g^{\mu \nu}=e_{a}^{\mu} e_{b}^{\nu} \eta^{a b}, \\
\gamma^{\mu}(x)=e_{a}^{\mu}(x) \gamma^{a},
\end{gathered}
$$

where $\gamma^{a}$ are constant matrices. Additionally, one can write

$$
S^{\lambda \nu}=\frac{1}{2}\left[\gamma^{\lambda}(x), \gamma^{\nu}(x)\right]
$$

The fermions have only one spin polarization in $(2+1)$ dimensions; then, the Dirac matrices can be expressed in terms of the Pauli spin matrices $\gamma^{i}=\left(\sigma^{3}, i \sigma^{1}, i \sigma^{2}\right)$ and they satisfy the anticommutation relation which is

$$
\left\{\sigma^{i}, \sigma^{j}\right\}=2 \eta^{i j} I_{2 \times 2}
$$

where $\eta^{i j}$ is the $(2+1)$-dimensional Minkowski space-time metric and $I_{2 \times 2}$ is the identity matrix. These matrices can be chosen as $\gamma^{0^{\dagger}}=-\gamma^{0}, \gamma^{i^{\dagger}}=\gamma^{i}$. If we use (4)-(7), we arrive at

$$
\begin{aligned}
& \Gamma_{0}=0, \\
& \Gamma_{1}=-\frac{1}{2} \cosh \tau \gamma^{0} \gamma^{1}, \\
& \Gamma_{2}=-\frac{1}{2}\left(\cosh \tau \sin \theta \gamma^{0} \gamma^{2}+\cos \theta \gamma^{1} \gamma^{2}\right) .
\end{aligned}
$$

Then, (1) becomes

$$
\begin{gathered}
\left(\gamma^{0}\left(\partial_{\tau}+\operatorname{coth} \tau\right)+i M \ell \widehat{E}+\frac{\gamma^{1}}{\sinh \tau}\left(\partial_{\theta}+\frac{1}{2} \cot \theta\right)\right. \\
\left.+\frac{\gamma^{2}}{\sinh \tau \sin \theta} \partial_{\phi}+i e \ell A_{1}(\tau, \theta) \gamma^{1}\right) \Psi=0
\end{gathered}
$$


It is noted that $\Psi$ depends on $(\tau, \theta, \phi)$ with two components, $\Psi=\left(\begin{array}{l}\psi_{1} \\ \psi_{2}\end{array}\right)$. Thus, (9) turns into

$$
\begin{aligned}
& \left(\frac{\partial}{\partial \tau}+\operatorname{coth} \tau+i M \ell\right) \psi_{1} \\
& \quad+\frac{i}{\sinh \tau}\left(\frac{\partial}{\partial \theta}+\frac{\cot \theta}{2}-\frac{i}{\sin \theta} \frac{\partial}{\partial \phi}+i e A_{1}(\tau, \theta)\right) \psi_{2} \\
& \quad=0 \\
& -\left(\frac{\partial}{\partial \tau}+\operatorname{coth}-i M \ell\right) \psi_{2} \\
& \quad+\frac{i}{\sinh \tau}\left(\frac{\partial}{\partial \theta}+\frac{\cot \theta}{2}+\frac{i}{\sin \theta} \frac{\partial}{\partial \phi}+i e A_{1}(\tau, \theta)\right) \psi_{1} \\
& \quad=0
\end{aligned}
$$

Applying the separation of variables process leads to

$$
\begin{aligned}
& \left(\frac{\partial}{\partial \tau}+\operatorname{coth} \tau+i M \ell\right) T_{1}(\tau)-\frac{i \omega_{2}}{\sinh \tau} T_{2}(\tau)=0 \\
& -\left(\frac{\partial}{\partial \tau}+\operatorname{coth} \tau-i M \ell\right) T_{2}(\tau)+\frac{i \omega_{1}}{\sinh \tau} T_{1}(\tau)=0 \\
& \left(-\frac{\partial}{\partial \theta}-\frac{\cot \theta}{2}+\frac{i}{\sin \theta} \frac{\partial}{\partial \phi}-i e A_{1}\right) Y_{2}(\theta, \phi) \\
& =\omega_{2} Y_{1}(\theta, \phi), \\
& \left(\frac{\partial}{\partial \theta}+\frac{\cot \theta}{2}+\frac{i}{\sin \theta} \frac{\partial}{\partial \phi}+i e A_{1}\right) Y_{1}(\theta, \phi) \\
& =\omega_{1} Y_{2}(\theta, \phi) .
\end{aligned}
$$

Here, $\psi_{1}=T_{1}(\tau) Y_{1}(\theta, \phi)$ and $\psi_{2}=T_{2}(\tau) Y_{2}(\theta, \phi)$ and $A_{\theta}(\tau, \theta)$ is chosen as $A_{\theta}(\tau, \theta)=\sinh \tau A_{1}(\theta)$, and $\omega_{1,2}$ are the separation constants. The angular part is defined as

$$
\left(\begin{array}{c}
Y_{1}(\theta, \phi) \\
Y_{2}(\theta, \phi)
\end{array}\right)=e^{i m \phi}\left(\begin{array}{c}
\Theta_{1}(\theta) \\
\Theta_{2}(\theta)
\end{array}\right)
$$

then we have

$$
\begin{gathered}
\left(-\frac{\partial}{\partial \theta}-\frac{\cot \theta}{2}-\frac{m}{\sin \theta}-i e A_{1}\right) \Theta_{2}=\omega \Theta_{1} \\
\left(\frac{\partial}{\partial \theta}+\frac{\cot \theta}{2}-\frac{m}{\sin \theta}+i e A_{1}\right) \Theta_{1}=\omega \Theta_{2} .
\end{gathered}
$$

We have used $\omega_{1}=\omega_{2}=\omega$. The first order angular and time dependent equations give us

$$
\begin{aligned}
- & \frac{d^{2} \Theta_{1}(\theta)}{d \theta^{2}}+\left(-2 i e A_{1}-\cot \theta\right) \frac{d \Theta_{1}(\theta)}{d \theta} \\
& +\left(\left(e A_{1}-\frac{i \cot \theta}{2}\right)^{2}-m \cot \theta \csc \theta\right. \\
& \left.+\left(m^{2}+\frac{1}{2}\right) \csc ^{2} \theta-i e \frac{\partial A_{1}}{\partial \theta}\right) \Theta_{1}(\theta)(\theta)=\omega^{2} \Theta_{1}(\theta), \\
- & \frac{d^{2} \Theta_{2}(\theta)}{d \theta^{2}}+\left(-2 i e A_{\theta}-\cot \theta\right) \frac{d \Theta_{2}(\theta)}{d \theta} \\
& +\left(\left(e A_{\theta}-\frac{i \cot \theta}{2}\right)^{2}+m \cot \theta \csc \theta\right. \\
& \left.+\left(m^{2}+\frac{1}{2}\right) \csc ^{2} \theta-i e \frac{\partial A_{1}}{\partial \theta}\right) \Theta_{2}(\theta)(\theta)=\omega^{2} \Theta_{2}(\theta) .
\end{aligned}
$$

Hence, the time dependent equations are obtained as

$$
\begin{aligned}
& \frac{d^{2} T_{1}(\tau)}{d \tau^{2}}+3 \operatorname{coth} \tau \frac{d T_{1}(\tau)}{d \tau}+(i \ell M \operatorname{coth} \tau \\
& \left.\quad+\left(\omega^{2}-1\right) \operatorname{csch} \tau^{2}+\ell^{2} M^{2}+2 \operatorname{coth} \tau^{2}\right) T_{1}(\tau)=0, \\
& \frac{d^{2} T_{2}(\tau)}{d \tau^{2}}+3 \operatorname{coth} \tau \frac{d T_{2}(\tau)}{d \tau}+(-i \ell M \operatorname{coth} \tau \\
& \left.\quad+\left(\omega^{2}-1\right) \operatorname{csch} \tau^{2}+\ell^{2} M^{2}+2 \operatorname{coth} \tau^{2}\right) T_{2}(\tau)=0 .
\end{aligned}
$$

\section{Exact Solutions}

Let us see the bound states and corresponding solutions of the Dirac Hamiltonian.

3.1. Solutions of Angular Part. In order to obtain a Schrödinger-like equation, we choose $A_{1}(\theta)=i(\cot \theta / 2 e)$ and use in (14); then we get

$$
\begin{aligned}
& V_{+}(\theta)=-m \cot \theta \csc \theta+m^{2} \csc ^{2} \theta \\
& V_{-}(\theta)=m \cot \theta \csc \theta+m^{2} \csc ^{2} \theta
\end{aligned}
$$

where $V_{1}$ and $V_{2}$ are the functions of the Hamiltonians which are

$$
\begin{aligned}
h_{+} \Theta_{1} & =\omega^{2} \Theta_{1}, \\
h_{-} \Theta_{2} & =\omega^{2} \Theta_{2}, \\
h_{ \pm} & =-\frac{d^{2}}{d \theta^{2}}+V_{ \pm}(\theta) .
\end{aligned}
$$


The partner Hamiltonians can be factorized as

$$
\begin{aligned}
& h_{-}=\mathbf{A}^{\dagger} \mathbf{A}, \\
& h_{+}=\mathbf{A A}^{\dagger}, \\
& \mathbf{A}=\frac{d}{d \theta}+W(\theta), \\
& \mathbf{A}^{\dagger}=-\frac{d}{d \theta}+W(\theta) .
\end{aligned}
$$

In our case superpotential $W(\theta)=B \csc \theta-A \cot \theta, A, B$ are constants, and $V_{ \pm}(\theta)=W(\theta)^{2} \mp \partial_{\theta} W$. It is known that (16) are shape invariant potentials when $m \rightarrow-m$ used in (16) can be obtained. If the supersymmetry is unbroken, the ground state of $h_{+}$has zero energy $\omega_{+, 0}=0$. The energy eigenvalues of the partner Hamiltonians are connected by operators in (19). Thus, we have

$$
\omega_{+, n}=\omega_{-, n-1}, \quad n=0,1, \ldots
$$

The eigenfunctions are related by the operators as

$$
\Theta_{-, n-1}=\frac{1}{\sqrt{\omega_{+, n}}} \mathbf{A} \Theta_{+, n}
$$

In [19], the potential is type $(P I)$ given below

$$
\begin{aligned}
V_{+}(\theta)= & -A^{2}+\left(A^{2}+B^{2}-A\right) \csc \theta^{2} \\
& -B(2 A-1) \csc \theta \cot \theta
\end{aligned}
$$

The solutions are given in [19] for (22) which are

$$
\begin{aligned}
& \omega_{+, n}=\omega_{-, n-1}= \pm \sqrt{(A+n)^{2}-A^{2}} \\
& \Theta_{+, n}=\Theta_{-, n}=N(1-\cos \theta)^{(A-B) / 2}(1+\cos \theta)^{(A+B) / 2} \\
& \cdot P_{n}^{(A-B-1 / 2 ; A+B+1 / 2)}(\cos \theta),
\end{aligned}
$$

where $P_{a}^{(b, c)}(x)$ stand for the Jacobi polynomials. For our case,

$$
\begin{aligned}
& A=\frac{1+2 m}{2}, \\
& B=\frac{1}{2} .
\end{aligned}
$$

The normalization constant $N$ is given by

$$
N=\frac{2^{m+2}}{2 n+m+2} \frac{\Gamma(n+m+3 / 2) \Gamma(n+3 / 2)}{n ! \Gamma(n+m+2)} \text {. }
$$

And the angular part solutions are

$$
\left(\begin{array}{c}
Y_{1}(\theta, \phi) \\
Y_{2}(\theta, \phi)
\end{array}\right)=e^{i m \phi}\left(\begin{array}{c}
\Theta_{-, n-1} \\
\Theta_{+, n}
\end{array}\right) .
$$

3.2. Solutions of the Time Dependent Part. Using a mapping that is

$$
T_{1,2}(\tau)=\operatorname{csch} \tau^{3 / 2} y_{1,2}(\tau)
$$

in (15), we get

$$
\begin{aligned}
& -\frac{d^{2} y_{1}}{d \tau^{2}} \\
& \quad+\left(\frac{1}{4}-\ell^{2} M^{2}-i \ell M \operatorname{coth} \tau-\left(\omega^{2}+\frac{1}{4}\right) \operatorname{csch} \tau^{2}\right) \\
& \quad \cdot y_{1}(\tau)=0 \\
& -\frac{d^{2} y_{2}}{d \tau^{2}} \\
& \quad+\left(\frac{1}{4}-\ell^{2} M^{2}+i \ell M \operatorname{coth} \tau-\left(\omega^{2}+\frac{1}{4}\right) \operatorname{csch} \tau^{2}\right) \\
& \quad \cdot y_{2}(\tau)=0 .
\end{aligned}
$$

Now that we have $z=i \operatorname{coth}(i \tau)(-\infty<\tau<\infty)$ in the above equations, we get

$$
\begin{aligned}
-(1+ & \left.z^{2}\right) \frac{d^{2} y_{k}}{d z^{2}}-2 z \frac{d y_{k}}{d z} \\
& +\left(-\frac{\ell^{2} M^{2}-1 / 4}{1+z^{2}}+\omega^{2}+\frac{1}{4}+\frac{\ell M \epsilon z}{1+z^{2}}\right) y_{k}(z)
\end{aligned}
$$

$=0$,

where $-\infty<z<\infty$ and we use a more compact form for (29) and

$$
\epsilon= \begin{cases}-1, & k=1 \\ +1, & k=2\end{cases}
$$

Then, if we put $y_{k}(z)=\left(1+z^{2}\right)^{-1 / 4} \bar{y}_{k}(z)$ into (30), we obtain

$$
\begin{aligned}
-(1 & \left.+z^{2}\right) \frac{d^{2} \bar{y}_{k}}{d z^{2}}-z \frac{d \bar{y}_{k}}{d z} \\
& +\left(-\frac{\ell^{2} M^{2}}{1+z^{2}}+\omega^{2}+\frac{\ell M \epsilon z}{1+z^{2}}\right) \bar{y}_{k}(z)=0 .
\end{aligned}
$$

If we give a polynomial solution which is given below

$$
\bar{y}_{k}(z)=(z+i)^{-(1 / 2)(A+i B)}(z-i)^{-(1 / 2)(A-i B)} P(z),
$$

where $P(z)$ is the unknown polynomial, and substituting (33) into (32), we get

$$
\begin{aligned}
(1+ & \left.z^{2}\right) P^{\prime \prime}(z)+(z(1-2 A)-2 B) P^{\prime}(z) \\
& +\left(A^{2}-\omega^{2}\right) P(z)=0
\end{aligned}
$$

Here $A, B$ are constants and $P(z)$ is the so-called Romanovski polynomials [20-22] which are solutions of the differential equation given by

$$
\begin{aligned}
\left(1+x^{2}\right) & \frac{d^{2} R(x)}{d x^{2}}+(2 b x+a) \frac{d R(x)}{d x} \\
& -\bar{v}(\bar{v}-1+2 b) R(x)=0, \quad-\infty<x<\infty,
\end{aligned}
$$


where $\bar{v}$ is a quantum number $\bar{v}=0,1, \ldots$ and $R(x)=$ $R_{\bar{v}}^{(a, b)}(x)$. Here, we note that the constants $A, B$ are given by

$$
\begin{aligned}
& A=\frac{1}{16 \ell M \epsilon}\left(-8 \ell M \epsilon+4 \sqrt{2} \ell^{2} M^{2} \sqrt{\mathbf{a}_{1}-1-4 \ell^{2} M^{2}}\right. \\
&\left.+\sqrt{2}\left(1+\mathbf{a}_{1}\right) \sqrt{\mathbf{a}_{1}-1-4 \ell^{2} M^{2}}\right), \\
& B=\sqrt{\frac{\mathbf{a}_{1}-1}{8}-\frac{1}{2} \ell^{2} M^{2}}, \\
& \mathbf{a}_{1}=\sqrt{\left(1+4 \ell^{2} M^{2}\right)^{2}+16 \ell^{2} M^{2}}, \\
&(a, b)=\left(-2 B, \frac{1}{2}-A\right) .
\end{aligned}
$$

Then we have

$$
\begin{aligned}
& \bar{y}_{k, \nu}(z) \\
& \quad=(z+i)^{-(1 / 2)(A+i B)}(z-i)^{-(1 / 2)(A-i B)} R_{\bar{\nu}}^{(a, b)}(z) .
\end{aligned}
$$

We may give the normalization integral as

$$
\int_{-\infty}^{\infty}\left(1+z^{2}\right)^{b-1} e^{a \arctan z} R_{\bar{\nu}^{\prime}}^{(a, b)}(z) R_{\bar{\nu}}^{(a, b)}(z) d z=\delta_{\bar{\nu}}^{\overline{\nu^{\prime}}} .
$$

The solution of (32) gives

$$
A^{2}-\omega^{2}=-\bar{\nu}(\bar{\nu}-1+2 b)
$$

and, from (42) and (23), one can also obtain

$$
A^{2}+\left(m+\frac{1}{2}\right)^{2}-\left(m+n+\frac{1}{2}\right)^{2}+\bar{\nu}(\bar{\nu}-1+2 b)=0 .
$$

According to (36) and (43), one can express $\ell$ as $\ell_{\nu}$ in terms of quantum numbers. From (43), we get

$$
A=v \pm \sqrt{n+2 m n+n^{2}} .
$$

If we compare (44) and (36), we have

$$
\begin{aligned}
& n^{2}+2 m n+n=\frac{1}{2}, \\
& \nu=\frac{\sqrt{2}}{16 \ell M \epsilon} \sqrt{\mathbf{a}_{1}-1-4 \ell^{2} M^{2}}\left(\mathbf{a}_{1}+1+4 \ell^{2} M^{2}\right) .
\end{aligned}
$$

Using (46) one can find $\ell$ which is a function of $\nu$.

\section{Pseudosupersymmetry Framework}

Following the fundamental aspects of pseudo-Hermitian quantum mechanics may lead to an outline for understanding spectral aspects of the quantum system. In the light of pseudo-Hermitian generalization of supersymmetric quantum mechanics, one can construct an unknown nonHermitian Hamiltonian.

Definition 1. If $\mathfrak{H}_{ \pm}$are separable Hilbert spaces and an operator $L: \mathfrak{H}_{+} \rightarrow \mathfrak{H}_{-}$is defined and $\eta_{ \pm}: H_{ \pm} \rightarrow$ $H_{ \pm}$are linear operators which are generally Hermitian and invertible, then, the pseudoadjoint of this operator $L^{\ddagger}$ : $H_{-} \rightarrow H_{+}$is equal to $L^{\ddagger}=\eta_{+}^{-1} L^{\dagger} \eta_{-}$.
Definition 2. Let $\eta_{ \pm}=\eta$ which belongs to $\mathfrak{H}_{ \pm}=\mathfrak{H}$. $L$ is a pseudo-Hermitian operator if

$$
L^{\dagger}=\eta L \eta^{-1} \text {. }
$$

Definition 3. $\eta$ is an invertible operator which satisfies $\eta \mathrm{H}^{-1}=\mathrm{H}^{\dagger} ; \mathrm{H}$ is called pseudo-Hermitian Hamiltonian [1113]. The $\eta$ representation of the Hamiltonian is

$$
H_{\eta}=\eta H \eta^{-1}=H_{\eta}^{\dagger} .
$$

It is noted that the wave function $\psi$ is $\psi=\eta \phi$ and satisfies the wave equation given below

$$
\begin{aligned}
H \phi & =i \frac{d \phi}{d t}, \\
\hbar & =c=1 .
\end{aligned}
$$

And the scalar product reads

$$
\langle\xi, \phi\rangle_{\rho}=\langle\zeta, \psi\rangle=\int \xi^{\dagger} \rho \phi d \tau, \quad \rho=\eta^{2} .
$$

In [16], the authors constructed unique and self-conjugate Dirac Hamiltonians in gravitational fields using Schwinger gauge. They used an initial Hamiltonian and showed that the system of tetrad vectors remained the same in the $\eta$ representation. Then, $\eta$ is defined by [16]

$$
\eta=(-\mathbf{g})^{1 / 4}\left(-\mathbf{g}^{00}\right)^{1 / 4},
$$

where $\mathbf{g}^{00}$ is the time component of the metric tensor and $\mathbf{g}=$ $\operatorname{det}\left[g_{\mu \nu}\right]$. Now following the pseudosupersymmetric aspects of the system, $H$ is a linear and diagonalizable operator and $Q_{i}$ are linear operators (called supercharge operators), $i, j=$ $1,2, \ldots, N$, and $\tau$ is a grading operator defining the unitary involution

$$
\tau^{\dagger} \tau=\tau \tau^{\dagger}=\tau^{2}=1
$$

If $N=1$, we have only one supercharge operator $Q$ which satisfies the algebra [11-13]

$$
\begin{aligned}
Q^{2} & =\left(Q^{\ddagger}\right)^{2}=0, \\
\left\{Q, Q^{\ddagger}\right\} & =\delta_{i}^{j} H .
\end{aligned}
$$

According to the ordinary supersymmetric quantum mechanics, two-component realization of the system is

$$
\begin{aligned}
\tau & =\left(\begin{array}{cc}
1 & 0 \\
0 & -1
\end{array}\right), \\
Q & =\left(\begin{array}{ll}
0 & 0 \\
\eta & 0
\end{array}\right), \\
H & =\left(\begin{array}{cc}
H_{+} & 0 \\
0 & H_{-}
\end{array}\right), \\
\eta & =\left(\begin{array}{ll}
\eta_{+} & 0 \\
0 & \eta_{-}
\end{array}\right),
\end{aligned}
$$


where $H_{+}=\mathscr{L}_{1} \mathscr{L}_{2}$ and $H_{-}=\mathscr{L}_{2} \mathscr{L}_{1}$. In [23], an unknown non-Hermitian operator $H_{+}$is linked to the adjoint of its pseudosupersymmetric partner Hamiltonian $H_{-}$; that is,

$$
\eta H_{+}=H_{-}^{\dagger} \eta \text {. }
$$

It is noted that $H_{+}$is diagonalizable system admitting a complete biorthonormal system of eigenvectors. If $\eta_{1}, \eta_{2}$ are the intertwining operators such that

$$
\begin{aligned}
& \eta_{1} H_{+}=H_{-} \eta_{1}, \\
& \eta_{2} H_{-}=H_{-}^{\dagger} \eta_{2},
\end{aligned}
$$

it can be seen that operator $H_{+}$is $\widetilde{\eta}=\eta^{\dagger} \eta_{1}$ pseudo-Hermitian and $H_{-}$is $\left(\eta_{2}^{\dagger}\right)^{-1}$ pseudo-Hermitian, $\eta=\eta_{2} \eta_{1}$ [23], where supercharge operators satisfy $Q^{\ddagger}=\bar{\eta}^{-1} Q^{\dagger} \bar{\eta}$ and we give $\bar{\eta}$ and $\widetilde{H}$ instead of $\eta$ and $H$ in (54) as

$$
\begin{aligned}
\bar{\eta} & =\left(\begin{array}{cc}
\widetilde{\eta} & 0 \\
0 & \left(\eta_{2}^{\dagger}\right)^{-1}
\end{array}\right), \\
\widetilde{H} & =\left(\begin{array}{cc}
H_{+} & 0 \\
0 & H_{-}^{\dagger}
\end{array}\right) .
\end{aligned}
$$

If $H_{ \pm}(\tau)$ are time dependent pseudo-Hermitian Hamiltonians, then we have

$$
\begin{aligned}
& H_{-}^{\dagger}=\eta_{2} H_{-} \eta_{2}^{-1}+i \eta_{2}^{-1} \frac{\partial \eta_{2}}{\partial \tau} \\
& H_{-}=\eta_{1} H_{+} \eta_{1}^{-1}+i \eta_{1}^{-1} \frac{\partial \eta_{1}}{\partial \tau} \\
& H_{+}=\eta^{-1} H_{-}^{+} \eta-i \eta^{-1} \frac{\partial \eta}{\partial \tau}
\end{aligned}
$$

The adjoint of (60) may be put into (59); then one can obtain

$$
\eta^{\dagger} \eta_{1} H_{+}=H_{+}^{\dagger} \eta^{\dagger} \eta_{1}+i\left(\frac{\partial \eta^{\dagger}}{\partial \tau} \eta_{1}-\frac{\partial \eta_{1}}{\partial \tau} \eta^{\dagger}\right)
$$

Using (1), let us introduce the Hamiltonian as

$$
\begin{aligned}
H_{-}= & i M \ell \gamma^{0}-i \operatorname{coth} \tau-i \frac{\gamma^{0} \gamma^{1}}{\sinh \tau} \partial_{\theta}-i \frac{\gamma^{0} \gamma^{1}}{\sinh \tau} \frac{\cot \theta}{2} \\
& -i \frac{\gamma^{0} \gamma^{2}}{\sinh \tau \sin \theta} \partial_{\phi}-i e \ell A_{1}(\tau, \theta) \gamma^{0} \gamma^{1} .
\end{aligned}
$$

Thus, from (58) and (51), we get

$$
\begin{aligned}
H_{-}^{\dagger}= & i M \ell \gamma^{0}-i \frac{\gamma^{0} \gamma^{1}}{\sinh \tau} \partial_{\theta}-i \frac{\gamma^{0} \gamma^{2}}{\sinh \tau \sin \theta} \partial_{\phi} \\
& -i e \ell A_{1}(\tau, \theta) \gamma^{0} \gamma^{1},
\end{aligned}
$$

and we note that

$$
\begin{aligned}
\eta_{2} & =i \ell^{3 / 2} \sinh \tau \sqrt{\sin \theta}, \\
\eta_{2}^{-1} \frac{\partial \eta_{2}}{\partial \tau} & =\operatorname{coth} \tau .
\end{aligned}
$$

Let us introduce a form for the metric operator $\eta_{1}$ as

$$
\eta_{1}=\ell^{2}(\sinh \tau)^{a_{1}}(\sqrt{\sin \theta})^{a_{2}}(\sqrt{\sin \phi})^{a_{3}},
$$

where $a_{1}, a_{2}$, and $a_{3}$ are real numbers. We may give the unknown Hamiltonian $H_{+}$as

$$
\begin{aligned}
H_{+}= & i M \ell \gamma^{0}-i \frac{\gamma^{0} \gamma^{1}}{\sinh \tau} \partial_{\theta}-i \frac{\gamma^{0} \gamma^{2}}{\sinh \tau \sin \theta} \partial_{\phi} \\
& -i e \ell A_{1}(\tau, \theta) \gamma^{0} \gamma^{1}+\gamma^{0} \gamma^{1} f[\tau, \theta, \phi] \\
& +\gamma^{0} \gamma^{2} g[\tau, \theta, \phi]+U(\tau) .
\end{aligned}
$$

Here $f[\tau, \theta, \phi], g[\tau, \theta, \phi], U(\tau)$ are the unknown functions. Now, both (59) and (60) lead to the following expressions:

$$
\begin{aligned}
\gamma^{0} \gamma^{1}\left(\frac{1}{2} i \cot \theta \csc h \tau\left(1+a_{2}\right)+f(\tau, \theta, \phi)\right) & =0, \\
\gamma^{0} \gamma^{2}\left(\frac{i a_{3}}{2} \cot \phi \csc \theta \csc h \tau+g(\tau, \theta, \phi)\right) & =0, \\
U(\tau)+i\left(a_{1}+1\right) \operatorname{coth} \tau & =0 .
\end{aligned}
$$

Choosing specific values such as $a_{1}=3 / 2, a_{2}=1$, and $a_{3}=1$, we obtain

$$
\begin{aligned}
H_{+}= & i M \ell \gamma^{0}-i \frac{\gamma^{0} \gamma^{1}}{\sinh \tau} \partial_{\theta}-i \frac{\gamma^{0} \gamma^{2}}{\sinh \tau \sin \theta} \partial_{\phi} \\
& -i e \ell A_{1}(\tau, \theta) \gamma^{0} \gamma^{1}-i \gamma^{0} \gamma^{1} \cot \theta \operatorname{csch} \tau \\
& -i \gamma^{0} \gamma^{2} \cot \phi \csc \theta \operatorname{csch} \tau-\frac{5 i}{2} \operatorname{coth} \tau .
\end{aligned}
$$

And the metric operator is given by

$$
\begin{aligned}
\eta_{1} & =\ell^{2}(\sinh \tau)^{3 / 2} \sqrt{\sin \theta} \sqrt{\sin \phi} \\
& =\left(-\mathbf{g}_{1}\right)^{1 / 4}\left(-\mathbf{g}_{1}^{00}\right)^{1 / 4} .
\end{aligned}
$$

This also shows that there may be a metric tensor for the Hamiltonian (68) which is the partner of $H_{-}$. We remind the reader that the metric tensor and metric operator for the system $H_{-}$are correspondingly given by

$$
\begin{aligned}
g_{\mu \nu} & =\left(\begin{array}{ccc}
\ell^{2} & 0 & 0 \\
0 & -\ell^{2} \sinh ^{2} \tau & 0 \\
0 & 0 & -\ell^{2} \sinh ^{2} \tau \sin ^{2} \theta
\end{array}\right), \\
\eta_{2} & =i \ell^{3 / 2} \sinh \tau \sqrt{\sin \theta} .
\end{aligned}
$$

Hence, we may obtain $\left(\mathbf{g}_{1}\right)_{\mu \nu}$ that may be the partner metric tensor of $g_{\mu \nu}$ as

$$
\left(\mathbf{g}_{1}\right)_{\mu \nu}
$$

$$
=\left(\begin{array}{cccc}
-\ell^{2} & 0 & 0 & 0 \\
0 & \ell^{2} \sinh ^{2} \tau & 0 & 0 \\
0 & 0 & \ell^{2} \sinh ^{2} \tau \sin ^{2} \theta & 0 \\
0 & 0 & 0 & \ell^{2} \sinh ^{2} \tau \sin ^{2} \phi
\end{array}\right) .
$$


Here, corresponding metric is

$$
\begin{aligned}
d s^{2}= & -\ell^{2} d \tau^{2}+\ell^{2} \sinh ^{2} \tau d \theta^{2}+\ell^{2} \sinh ^{2} \tau \sin ^{2} \theta d \phi^{2} \\
& +\ell^{2} \sinh ^{2} \tau \sin ^{2} \phi d \chi^{2}
\end{aligned}
$$

and this is the metric of the four-dimensional world in hyperspherical coordinates.

\section{Conclusions}

The Dirac operator is considered in a three-dimensional gravity and decomposed into time dependent and angular parts. After performing the separation of variables, the angular Dirac equation is reduced to the Klein-Gordon-like partner Hamiltonians possessing shape invariant trigonometric potentials whose real spectrum is given and solutions of the spinor wave functions are written in terms of the Jacobi polynomials. Thus, we have examined the de Sitter space-time metric solutions of the Dirac equation where the Hamiltonian becomes a non-Hermitian one because of this time dependency of the metric. On the other hand, the time dependent part of the Dirac system (29) has a time dependent potential function whose form is similar to the complex Eckart potential in the literature [24]. However, the function $\csc h^{2} \tau$ has a wrong sign in our case because we have used an appropriate mapping to obtain a soluble hypergeometric differential equation and we have obtained the solutions in terms of the Romanovski polynomials. According to the theory of non-Hermitian Hamiltonians in quantum physics, a subclass of non-Hermitian operators is the pseudo-Hermitian operators which satisfy the $\eta$ inner product. Accordingly, pseudo-Hermiticity is fulfilled by a weight operator which was shown by Parker earlier and, using the initial Hamiltonian that becomes a pseudo-Hermitian Hamiltonian in the view of a time dependent metric, we have obtained real spectrum and a condition on quantum numbers at the end of the exact solutions. The radius of the universe is given by $\ell$ and we have seen that this radius depends on $v$ quantum number. Because two partner Hamiltonians are pseudo-Hermitian, we have found the metric operator $\eta_{1}$ that links $H_{ \pm}$. We have also seen that another metric tensor for the partner Hamiltonian $H_{+}$may be obtained using pseudosupersymmetry. Moreover, we have shown that a new $(3+1)$-dimensional Hamiltonian $H_{+}$can be obtained by means of the pseudosupersymmetric procedure which means that one can expand $(2+1)$ Dirac system to $(3+1)$ dimensions. In our case, the metric operators $\eta_{1}$ and $\eta_{2}$ are not differential operators. We have used an ansatze for the $\eta_{1}$ metric operator, however, if there is a symmetry in curved Dirac systems which gives metric operator without giving an ansatze that can be searched in future works. This study also aims to attract the interest of both the readers of nonHermitian Hamiltonian systems and the quantum gravity to the area which is an intersection of those theories.

\section{Conflict of Interests}

The author declares that there is no conflict of interests regarding the publication of this paper.

\section{References}

[1] S. A. Werner, J. L. Staudenmann, and R. Colella, "Effect of Earth's rotation on the quantum mechanical phase of the neutron," Physical Review Letters, vol. 42, no. 17, pp. 1103-1106, 1979.

[2] A. Albrecht, A. Retzker, and M. B. Plenio, "Testing quantum gravity by nanodiamond interferometry with nitrogen-vacancy centers," Physical Review A, vol. 90, no. 3, Article ID 033834, 2014.

[3] I. R. Berchera, I. P. Degiovanni, S. Olivares, and M. Genovese, "Quantum light in coupled interferometers for quantum gravity tests," Physical Review Letters, vol. 110, no. 21, Article ID 213601, 2013.

[4] L. Parker, "One-electron atom as a probe of spacetime curvature," Physical Review D, vol. 22, no. 8, pp. 1922-1934, 1980.

[5] M. A. Jafarizadeh and S. K. Moayedi, "Degeneracy and parasupersymmetry of dirac hamiltonian in $(2+1)$-space-time," Journal of Mathematical Physics, vol. 40, no. 9, pp. 4274-4289, 1999.

[6] Y. Sucu and N. Ünal, "Exact solution of Dirac equation in $2+1$ dimensional gravity," Journal of Mathematical Physics, vol. 48, no. 5, Article ID 052503, 2007.

[7] Ö. Gürtuğ, M. Halilsoy, and S. Habib Mazharimousavi, “Quantum probes of timelike naked singularities in 2+1-dimensional power-law spacetimes," Advances in High Energy Physics, vol. 2015, Article ID 684731, 10 pages, 2015.

[8] S. M. Morsink and R. B. Mann, "Black hole radiation of Dirac particles in $1+1$ dimensions," Classical and Quantum Gravity, vol. 8, no. 12, pp. 2257-2268, 1991.

[9] Ö. Açık, Ü. Ertem, M. Önder, and A. Verçin, "First-order symmetries of the Dirac equation in a curved background: a unified dynamical symmetry condition," Classical and Quantum Gravity, vol. 26, no. 7, Article ID 075001, 2009.

[10] C. M. Bender and S. Boettcher, "Real spectra in non-hermitian Hamiltonians having $\mathscr{P} \mathscr{T}$ symmetry," Physical Review Letters, vol. 80 , no. 24, pp. 5243-5246, 1998.

[11] A. Mostafazadeh, "Pseudo-Hermiticity versus PT symmetry: the necessary condition for the reality of the spectrum of a nonHermitian Hamiltonian," Journal of Mathematical Physics, vol. 43, no. 1, pp. 205-214, 2002.

[12] A. Mostafazadeh, "Pseudo-Hermiticity versus PT-symmetry. II. a complete characterization of non-Hermitian Hamiltonians with a real spectrum," Journal of Mathematical Physics, vol. 43, no. 5, pp. 2814-2816, 2002.

[13] A. Mostafazadeh, "Pseudo-hermiticity versus PT-symmetry III: equivalence of pseudo-Hermiticity and the presence of antilinear symmetries," Journal of Mathematical Physics, vol. 43, no. 8, pp. 3944-3951, 2002.

[14] X. Huang and L. Parker, "Hermiticity of the Dirac Hamiltonian in curved spacetime," Physical Review D, vol. 79, no. 2, Article ID 024020, 2009.

[15] M. V. Gorbatenko and V. P. Neznamov, "Solution of the problem of uniqueness and Hermiticity of Hamiltonians for Dirac particles in gravitational fields," Physical Review D, vol. 82, no. 10, Article ID 104056, 2010. 
[16] M. V. Gorbatenko and V. P. Neznamov, "Uniqueness and selfconjugacy of Dirac Hamiltonians in arbitrary gravitational fields," Physical Review D, vol. 83, no. 10, Article ID 105002, 2011.

[17] A. Iorio, "Curved spacetimes and curved graphene: a status report of the Weyl symmetry approach," International Journal of Modern Physics D. Gravitation, Astrophysics, Cosmology, vol. 24, no. 5, Article ID 1530013, 2015.

[18] A. Corichi and A. Gomberoff, "On a spacetime duality in $2+1$ gravity," Classical and Quantum Gravity, vol. 16, no. 11, pp. 35793598, 1999.

[19] G. Lévai, "A search for shape-invariant solvable potentials," Journal of Physics A: Mathematical and General, vol. 22, no. 6, pp. 689-702, 1989.

[20] D. E. Alvarez-Castillo and M. Kirchbach, "The real exact solutions to the hyperbolic scarf potential," Revista Mexicana de Física E, vol. 53, no. 2, pp. 143-154, 2007.

[21] C. Quesne, "Extending Romanovski polynomials in quantum mechanics," Journal of Mathematical Physics, vol. 54, no. 12, Article ID 122103, 2013.

[22] G.-H. Sun, S.-H. Dong, and N. Saad, "Quantum information entropies for an asymmetric trigonometric Rosen-Morse potential," Annalen der Physik, vol. 525, no. 12, pp. 934-943, 2013.

[23] R. Roychoudhury and B. Roy, "Intertwining operator in nonlinear pseudo-supersymmetry," Physics Letters A, vol. 361, no. 4-5, pp. 291-293, 2007.

[24] C.-S. Jia, S.-C. Li, Y. Li, and L.-T. Sun, "Pseudo-Hermitian potential models with $P T$ symmetry," Physics Letters A, vol. 300, no. 2-3, pp. 115-121, 2002. 

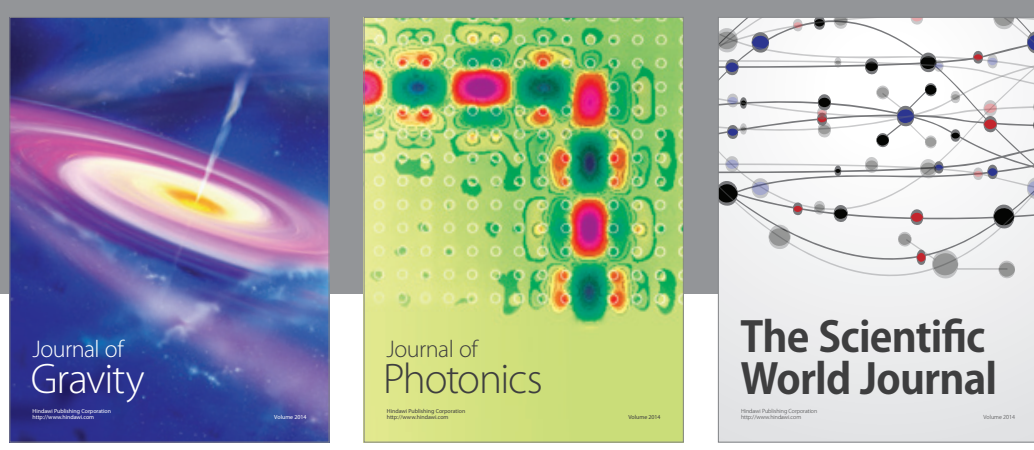

The Scientific World Journal
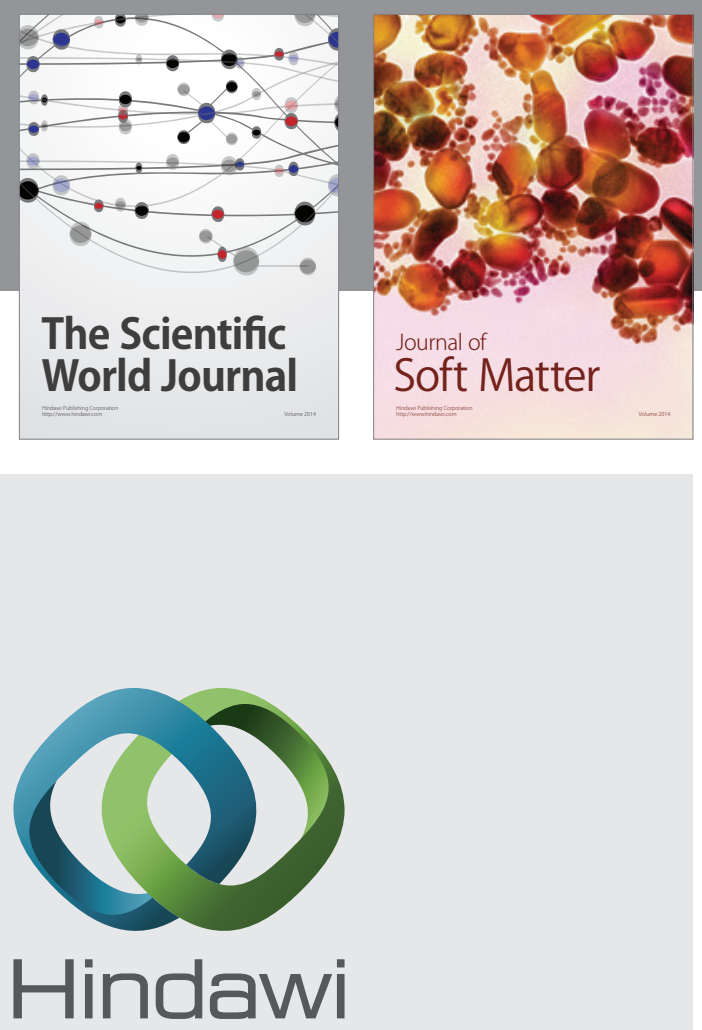

Submit your manuscripts at

http://www.hindawi.com

nternational Journal of

Statistical Mechanics
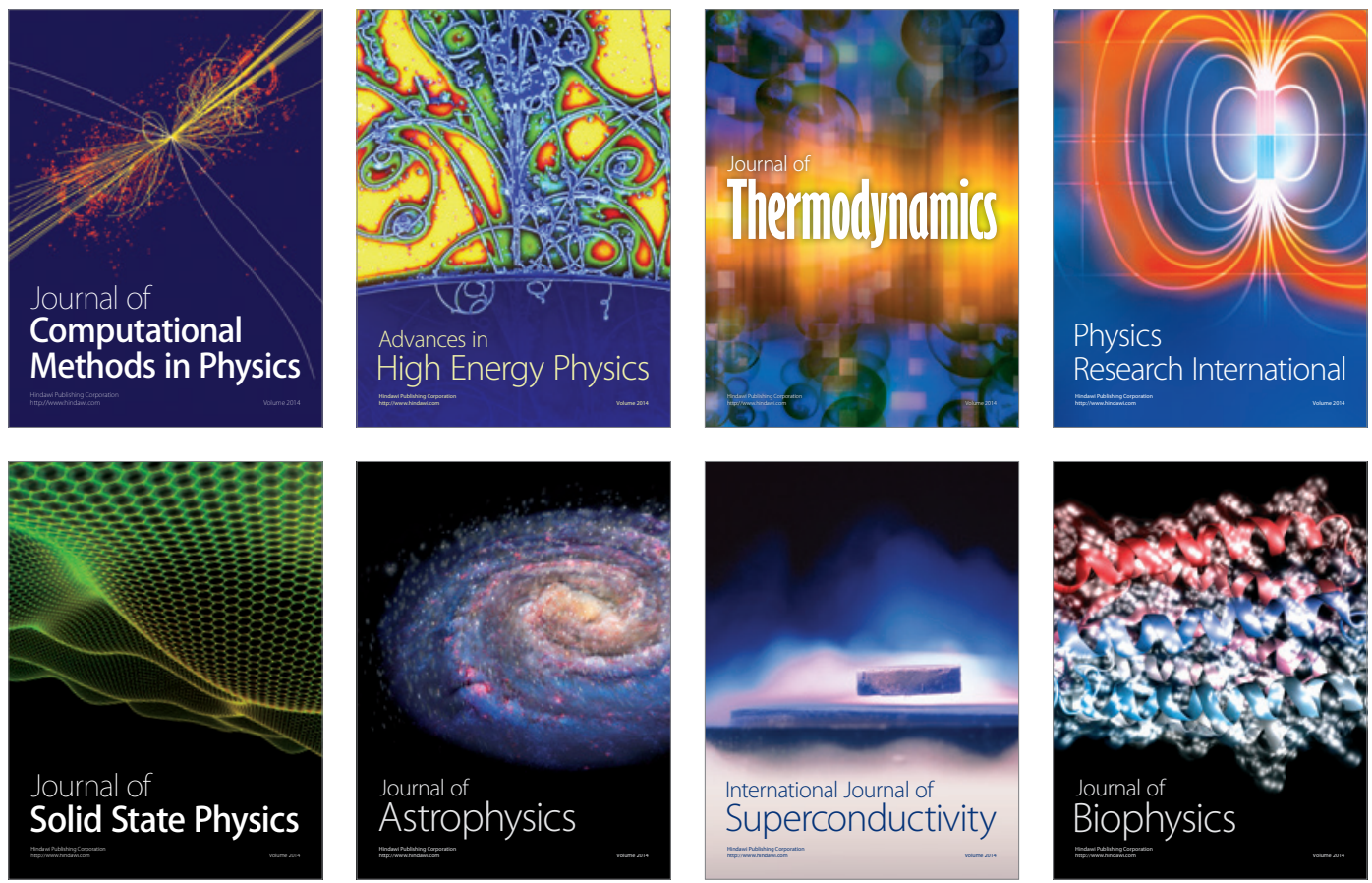
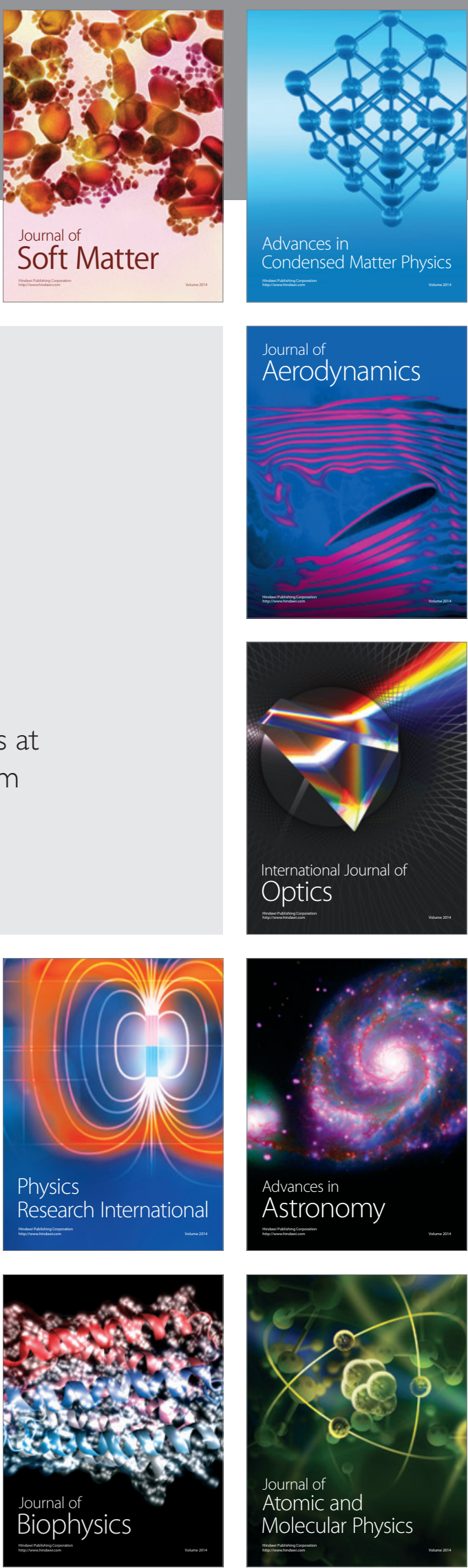